\title{
Factores que influyen en la integración del Programa de Inclusión y Alfabetización Digital en la docencia en escuelas primarias
}

\section{Factors influencing the Integration of the Digital Literacy and Inclusion Program into Primary School Teaching}

\author{
Jesús Alfonso Beltrán-Sánchez (1) jbeltrans@tec.mx \\ Ramona Imelda García López (2) imelda.garcia@itson.edu.mx \\ María Soledad Ramírez-Montoya (1) solramirez@tec.mx \\ Jesús Tánori Quintana (2) jesus.tanori@itson.edu.mx \\ (1) Tecnológico de Monterrey \\ (2) Tecnológico de Sonora \\ (Recibido: 18 de julio de 2017; Aceptado para su publicación: 3 de octubre de 2017)
}

Cómo citar: Beltrán-Sánchez, J. A., García, R. I., Ramírez-Montoya, M. S. y Tánori, J. (2019). Factores que influyen en la integración del Programa de Inclusión y Alfabetización Digital en la docencia en escuelas primarias. Revista Electrónica de Investigación Educativa, 21, e31, 1-11. doi:10.24320/redie.2019.21.e31.2088

\section{Resumen}

La presente investigación pretende identificar los factores relacionados en la integración del Programa de Inclusión y Alfabetización Digital en las actividades del docente de educación primaria pública en México; para ello, se utilizó una metodología cuantitativa de alcance explicativo con una muestra de 203 docentes de un municipio del sur del estado de Sonora. Fueron medidos cuatro factores resultantes de la revisión teórica (escolares, personales, programáticos y contextuales), los resultados demuestran que las variables asociadas al factor personal del profesor son aquellas que logran explicar e impactar en mayor medida la integración del programa. Se concluye la presencia de una necesidad manifiesta en cuanto a la reformulación y desarrollo de programas de capacitación y concientización sobre el impacto de la tecnología y los beneficios que conlleva para el aprendizaje.

Palabras clave: Alfabetización informacional, docente de escuela primaria, tecnología educacional, actitud del docente.

\section{Abstract}

The present investigation aims to identify the factors related to the integration of the Inclusion and Digital Literacy Program (IDLP or PIAD in Spanish) in the activities carried out by elementary school teachers in Mexican public institutes. To that end, a quantitative methodology with an explanatory scope was used, along with a sample size of 203 teachers from a county south of the state of Sonora, Mexico. Four remaining factors from the theoretical review (educative, personal, pragmatic and contextual) were measured and the results obtained show that the variables associated to the personal factor of the professor are those that are able to explain and impact to a greater degree the integration of the program. It concludes the presence of a manifested necessity in regards to the reformulation and development of capacitation programs and awareness over the impact of technology and the benefits that it brings with the purpose of accomplishing the learning process. 


\section{Introducción}

En México, la Secretaría de Educación Pública (SEP) ha realizado diversos esfuerzos por integrar las Tecnologías de la Información y la Comunicación (TIC) en la educación básica con programas tales como: COEEBA (Computación Electrónica en Educación Básica), la propuesta "Red escolar", el programa "Enciclomedia" y "Habilidades digitales para todos" (Martínez y Cabello, 2012), predecesores del programa federal "Mi CompuMx" que inició en el 2013 y hoy es el Programa de Inclusión y Alfabetización Digital (PIAD) (SEP, 2013).

El PIAD basa sus principios de aplicación en iniciativas como One Laptop per Child (OLPC), desarrollada por Nicholas Negroponte y Seymour Papert (OLPC, 2011), además del Plan Ceibal Uruguayo (Plan Ceibal, 2011); dichos programas tienen como sustento teórico al paradigma construccionista, cabe señalar que son programas sin fines de lucro y su principal actividad consiste en la entrega de computadoras a niños de educación primaria con miras a reducir la brecha digital y crear una cultura de ciudadanía digital.

En México, el PIAD tiene diversos matices, además de los ya mencionados, puesto que la entrega de computadoras se hace exclusivamente a niños de 50. y 60. sexto de primaria, así como a profesores, directivos y administrativos, entre otros. La entrega se realizó a través de una fase piloto en la cual fueron seleccionados tres estados del país por su pluralidad económica, política y cultural, en este caso Colima, Sonora y Tabasco (Staff Presidencia, 2013); en primera instancia se hizo una entrega cerca de 220,000 equipos en todas las escuelas públicas de las entidades mencionadas (SEP, 2013).

La inclusión de las TIC en la educación siempre ha constituido un rubro pendiente para los países en desarrollo, representando así un foco de análisis para los investigadores, quienes se han dado a la tarea de identificar cuáles son los factores más relevantes que influyen en la apropiación de la tecnología (De Pablos, Colás y González, 2009; Liu, 2011; Peeraer y Van Petegam, 2010; Rodríguez, García, Ibañez, González, y Heine, 2009; Trucco y Espejo, 2013; Valdés, Arreola, Angulo, Carlos y García, 2010); de los resultados de estas investigaciones se destaca la importancia de formar docentes con habilidades que permitan integrar la tecnología y la pedagogía, así como considerar la actitud del profesorado hacia las TIC.

A partir del 2014 un grupo de investigadores ha analizado el impacto de la entrega gratuita de equipos, haciendo énfasis en las experiencias de los actores que participan en el uso de los recursos del PIAD (Beltrán, García y Ramírez, 2015a; Beltrán, García y Ramírez, 2015b; Díaz, Rodríguez, Sánchez, Rivera y Ramírez, 2015; García, Angulo y Cuevas, 2015; Rivera, Mercado y Ramírez, 2015; Rocha y Ramírez, 2015). Entre los principales hallazgos de estos estudios destaca la falta de formación para docentes, poco conocimiento percibido por los profesores en cuanto a la integración de las TIC, falta de interés de diferentes actores, entre otros aspectos; en este sentido, cabe plantearse cuáles son los factores significativos que más influyen en la integración pedagógica de la tecnología en México.

\subsection{Factores que influyen en la integración tecnológica en las instituciones de educación}

Los cambios globales y la situación actual ponderan la necesidad de contar con ciudadanos mejor preparados, capaces de enfrentar los retos que la compleja e incierta realidad plantea (Morales, 2013). Afrontando los nuevos retos que caracterizan a la sociedad actual, diversos gobiernos han decidido modificar sus políticas en materia educativa, optando por trabajar en la integración de las Tıc en los planteles educativos (De Pablos et al., 2009; Peeraer y Van Petegem, 2010; Trucco y Espejo, 2013); a lo anterior se suma la necesidad de identificar los factores que están inmersos en la apropiación tecnológica de los recursos. En este sentido, Trucco y Espejo (2014) realizan una investigación en el marco del Plan CEIBAL y de los aspectos involucrados en los procesos de apropiación de los recursos tecnológicos por parte de los distintos beneficiarios del plan; dichos autores determinan una serie de factores divididos en cuatro categorías: de contexto, personales, programáticos y de escuela (ver figura 1). De éstos, el factor que no resultó estadísticamente relevante fue el contexto sociocultural, aunque los autores mencionan que eso tiene sentido puesto que no necesariamente importa dónde viva el profesor sino que atañe al estudiante por vivir en la zona. Cabe destacar que el nivel de significación los demás factores dentro de la categoría de contexto, oscila entre 0.46 y 0.89 , y se hace hincapié en considerar ciertos factores sociales, 
como el contexto socioeconómico del estudiante y el capital cultural asociado.

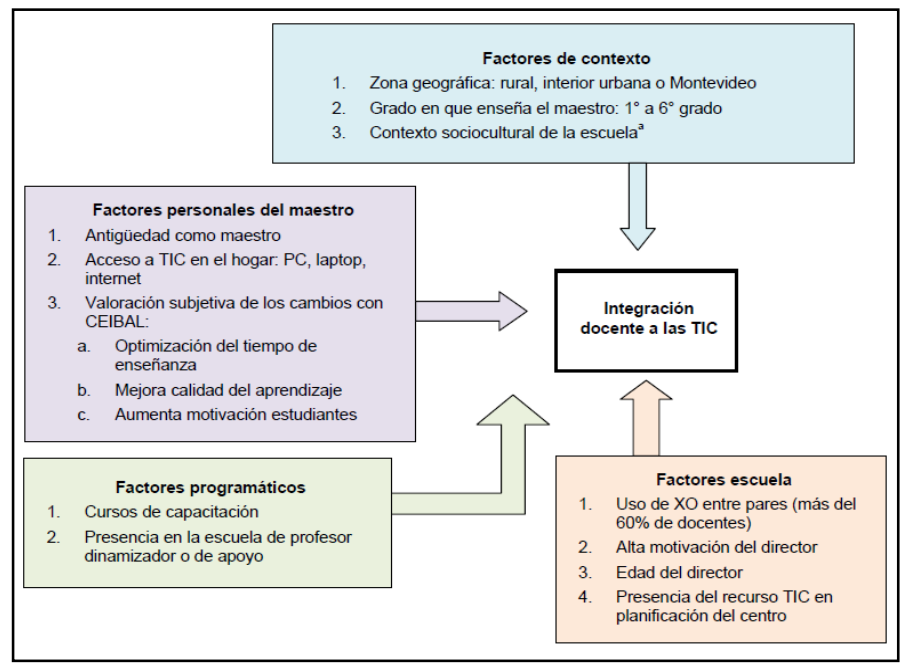

Figura 1. Factores asociados a la integración docente a las TIC

Desde otra perspectiva, Cabero et al. (2014) realizan una propuesta sobre los factores que permitirían al docente contar con un mejor Conocimiento Tecnológico Pedagógico del Contenido (TPACK, por sus siglas en inglés); en este sentido un docente con un mejor TPACK cuenta con un mejor nivel de apropiación debido a las dimensiones del mismo modelo. Los autores sugieren no dejar de lado los factores intangibles, como lo son la estructura organizativa, la autoeficacia percibida y los aspectos intangibles compuestos por las ideologías y aspectos éticos que marcan el currículum y su formación docente (ver figura 2).

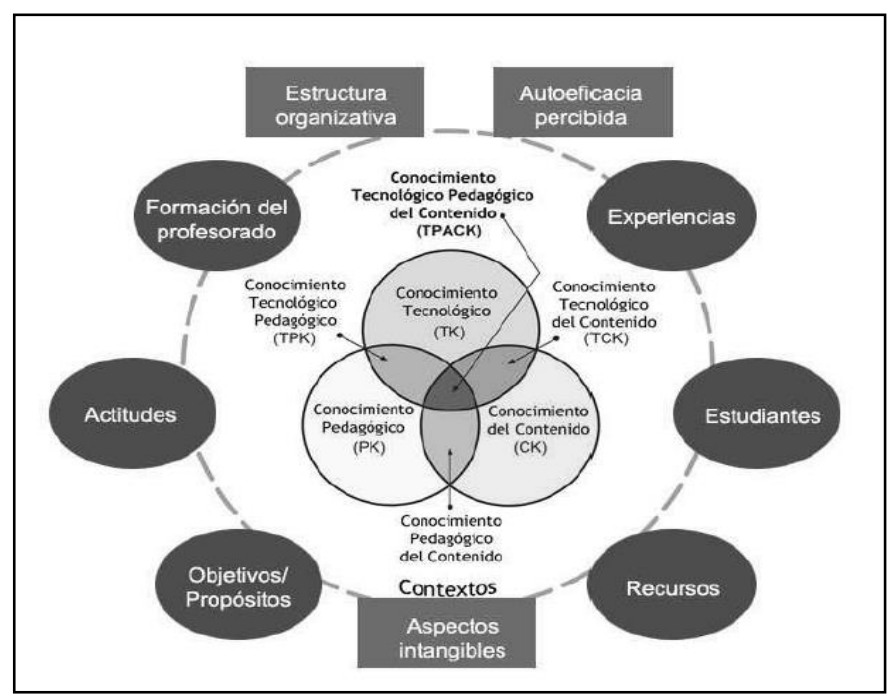

Figura 2. Factores que influyen en la integración del TPACK (Cabero et al. 2014)

\subsection{Modelo TPACK}

El modelo TPACK es propuesto por Mishra y Kohler (2006), quienes basaron su trabajo en las investigaciones de Shulman $(1986,1987)$, quien a su vez partió de la idea de que los docentes deben contar con conocimientos tanto de la disciplina como pedagógicos, por ello propuso el modelo PCK (Conocimiento Pedagógico del Contenido). 
El modelo TPACK explica los conocimientos necesarios del docente contemporáneo perteneciente a una sociedad regida por la tecnología. Este modelo propone tres conocimientos básicos: el pedagógico, el tecnológico y de contenido o disciplinar y la conjugación entre los mismos (ver tabla l y figura 3).

Tabla I. Descripción de los conocimientos que integran al TPACK

\begin{tabular}{|c|c|}
\hline Tipo de conocimiento & Descripción \\
\hline Conocimiento Pedagógico & $\begin{array}{l}\text { Conocimiento sobre las actividades pedagógicas generales, y de los procesos y } \\
\text { prácticas del método de enseñanza y cómo se relacionan con el pensamiento y los } \\
\text { propósitos educativos. }\end{array}$ \\
\hline Conocimiento Tecnológico & $\begin{array}{l}\text { Conocimiento que los profesores tienen respecto a cómo las diferentes } \\
\text { tecnologías se presentan para desarrollar su actividad profesional de la enseñanza. }\end{array}$ \\
\hline $\begin{array}{l}\text { Conocimiento } \\
\text { del Contenido }\end{array}$ & Conocimiento real que el profesorado tiene de aquello que debe ser la enseñanza. \\
\hline $\begin{array}{l}\text { Conocimiento } \\
\text { Tecnológico-Pedagógico }\end{array}$ & $\begin{array}{l}\text { Conocimiento de cómo las diversas tecnologías pueden ser utilizadas en la } \\
\text { enseñanza, y para comprender que el uso de la tecnología puede cambiar la forma } \\
\text { de enseñar de los profesores y de organizar la escenografía de la enseñanza. }\end{array}$ \\
\hline $\begin{array}{l}\text { Conocimiento Tecnológico } \\
\text { del Contenido }\end{array}$ & $\begin{array}{l}\text { Conocimiento de cómo la tecnología puede crear nuevas representaciones para } \\
\text { contenidos específicos. }\end{array}$ \\
\hline $\begin{array}{l}\text { Conocimiento Pedagógico } \\
\text { del Contenido }\end{array}$ & $\begin{array}{l}\text { Incluye la comprensión de las representaciones sobre temas específicos en una } \\
\text { disciplina determinada y cómo se podría utilizar como parte de las actividades de } \\
\text { enseñanza para promover el aprendizaje de los estudiantes. }\end{array}$ \\
\hline $\begin{array}{l}\text { Conocimiento } \\
\text { Tecnológico-Pedagógico } \\
\text { del Contenido }\end{array}$ & $\begin{array}{l}\text { Se refiere al conocimiento de un profesor sobre cómo coordinar el uso de las } \\
\text { actividades específicas de las materias o actividades sobre temas específicos (AT), } \\
\text { haciéndolo con representaciones sobre temas concretos, empleando las TIC para } \\
\text { facilitar el aprendizaje del estudiante. }\end{array}$ \\
\hline
\end{tabular}

Fuente: Cabero et al. (2014) y Mishra y Koehler (2006).

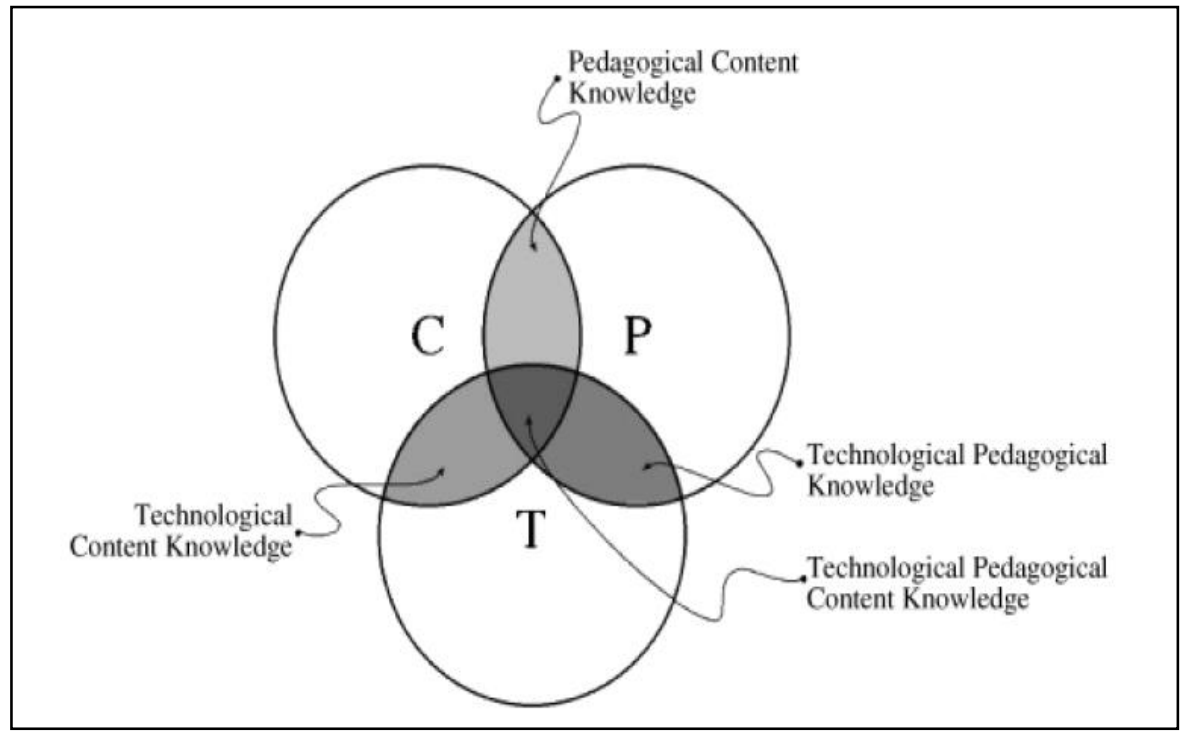

Fuente: Mishra y Koehler (2006).

Figura 3. Modelo del TPACK

Existen diversas investigaciones sobre la aplicación del modelo TPACK, desde evaluaciones sobre los conocimientos de los docentes, desarrollo de perfiles de formación inicial docente y el diseño y construcción de instrumentos válidos y confiables (Aguirre, 2014; Angeli y Valanides, 2009; Archambault y Barnett, 2010; Cabero et al., 2014; Kabakci et al., 2010; Lázaro, Gisbert, González y Camacho, 2014; Llorente, Romero, Román y Barroso, 2014; Nogueira, 2014; Sing, Ling, Tsai y Wee, 2011). Es debido a los resultados obtenidos y los precedentes del modelo que se toma como referencia en esta investigación. 


\section{Método}

Se presenta un estudio cuantitativo de tipo transversal con un alcance explicativo. Las investigaciones con alcances explicativos van más allá de la descripción de conceptos o fenómenos o del establecimiento de relaciones, puesto que están dirigidos a encontrar causas (Gómez, 2006); es decir, pretenden determinar una relación causa-efecto (González, Escoto y Chávez, 2017). En esta investigación se busca identificar los factores que se relacionan con la integración del programa (causas) y cuál es su aporte (efectos).

La población estuvo conformada por 494 docentes de escuelas primarias públicas de un municipio de la zona sur del estado de Sonora (México); todos impartiendo clases en 5o. y 6o. de primaria.

Se utilizó un muestreo no probabilístico intencional, donde el principal criterio de inclusión fue la disposición de los profesores en colaborar en el estudio. Del total de docentes 91 son mujeres y 112 son hombres con una edad media de 36.2 años (un mínimo de 21 y un máximo de 57 años); 94 profesores cuentan con licenciatura (46.3\%), 79 maestría (38.9) y 30 con doctorado (14.8\%). En cuanto a su interacción con la tecnología, 14 profesores reportó no tener computadora en casa (6.9\%) y 36 no cuenta con servicio de Internet (17.7); por último, 70 profesores (34.5\%) mencionan no haber recibidio capacitación en los últimos 3 años.

Los instrumentos utilizados en el estudios fueron tres encuestas para la medición de las variables: 1) TPACK, 2) competencia informacional y 3) la actitud del docente hacia el programa "Mi CompuMx". Cabe mencionar que estos instrumentos incluyen tanto datos de identificación como personales, de la escuela, programáticos y de contexto.

TPACK. El instrumento para medir este constructo se diseñó con base en la investigación realizada por Sing, Ling, Tsai y Wee (2011) quienes a su vez se basan en Schmidt et al. (2009), además de ser complementado por el estudio de Cabero et al. (2014).

Competencia Informacional. Se entiende por competencia informacional al conocimiento sobre la navegación en red, búsqueda, uso, producción y gestión de la información (Area, 2009). El instrumento para medir la competencia informacional fue diseñado con base en la investigación realizada por Rangel (2015).

Actitud docente hacia el programa "Mi CompuMx". El instrumento para medir la actitud docente hacia el programa "Mi CompuMx" es una adaptación del instrumento de Valdés, Arreola, Angulo, Carlos y García (2011).

\subsection{Propiedades métricas}

Para la validez de constructo se aplicó a cada uno de los instrumentos un análisis factorial exploratorio con el método de extracción de máxima verosimilitud y con rotación Oblimin, mientras que para determinar la confiabilidad se utilizó como índice de consistencia interna el alfa de Cronbach. Los resultados obtenidos (ver tabla 2) resultaron satisfactorios según los criterios establecidos por De Vellis (2012) y Field (2009). 
Tabla II. Descripción de los instrumentos y resultados obtenidos del análisis exploratorio y alfa de Cronbach

\begin{tabular}{|c|c|c|c|}
\hline & TPACK & $\begin{array}{l}\text { Competencia } \\
\text { informacional }\end{array}$ & $\begin{array}{c}\text { Actitud docente } \\
\text { hacia el programa }\end{array}$ \\
\hline Cantidad de ítems & 31 & 7 & 20 \\
\hline Escala & \multicolumn{3}{|c|}{ (0) Totalmente desacuerdo - (4) Totalmente de acuerdo } \\
\hline Consistencia interna (alfa de Cronbach) & $.870-.947$ & .889 & .836 y .937 \\
\hline Análisis exploratorio (KMO) & .925 & .838 & .922 \\
\hline Varianza explicada & $61.90 \%$ & $54.08 \%$ & $62.05 \%$ \\
\hline Cantidad de factores & 6 & 1 & 2 \\
\hline Ejemplo de ítem & $\begin{array}{l}\text { Tengo varios métodos } \\
\text { y estrategias para } \\
\text { desarrollar mi } \\
\text { conocimiento sobre } \\
\text { matemáticas. }\end{array}$ & $\begin{array}{l}\text { Identifico datos o } \\
\text { información relevante } \\
\text { y actual en sitios } \\
\text { reconocidos por la } \\
\text { comunidad científica. }\end{array}$ & $\begin{array}{l}\text { El programa mejora el } \\
\text { aprendizaje de los } \\
\text { estudiantes. }\end{array}$ \\
\hline
\end{tabular}

Para recolectar la información se pidió autorización a los directores de los planteles para explicarles los objetivos del estudio y obtener la información correspondiente; posteriormente se solicitó la cooperación voluntaria de los profesores de 50. y 6o. grado para que respondieran el instrumento diseñado, garantizando la confidencialidad de los resultados.

\section{Resultados}

\subsection{Niveles de conocimiento}

Como ya se mencionó, la escala de medición va de 0 (totalmente en descuerdo) a 4 (totalmente de acuerdo), en este sentido los puntajes más cercanos al 4 es indicativo de un nivel elevado respecto a la variable de medición, bajo esta premisa es posible decir que los conocimientos de los docentes relacionados con la tecnología son percibidos de regulares a buenos, y lo mismo sucede con la competencia informacional; sin embargo, los puntajes obtenidos en la variable actitudinal fueron bajos, como ilustra la tabla III.

Tabla III. Niveles de conocimiento de TPACK, competencia informacional y actitud hacia el programa

\begin{tabular}{l|c|c|c|c}
\hline Variables & M & DS & Máx. & Mín. \\
\hline Conocimiento del Contenido & 3.24 & .565 & 4 & 1 \\
Conocimiento Tecnológico & 2.73 & .684 & 4 & 1 \\
Conocimiento Pedagógico & 3.41 & .443 & 4 & 1 \\
Conocimiento del Tecnocontenido & 2.82 & .664 & 4 & 0 \\
Conocimiento Tecnopedagógico & 3.08 & .589 & 4 & 1 \\
Conocimiento Tecnológico-Pedagógico del Contenido & 2.95 & .618 & 4 & 1 \\
Informacional & 2.91 & .675 & 4 & 1 \\
Actitud hacia el programa & 2.64 & .645 & 4 & 0 \\
\hline
\end{tabular}

\subsection{Factores relacionados con la integración del programa}

Con el fin de identificar áreas de oportunidad se aplicó la prueba de regresión lineal donde se relacionan los puntajes obtenidos en las variables de TPACK, competencia informacional y la actitud de los profesores hacia el programa de inclusión y alfabetización tecnológica, como se ilustra en la figura 4. 


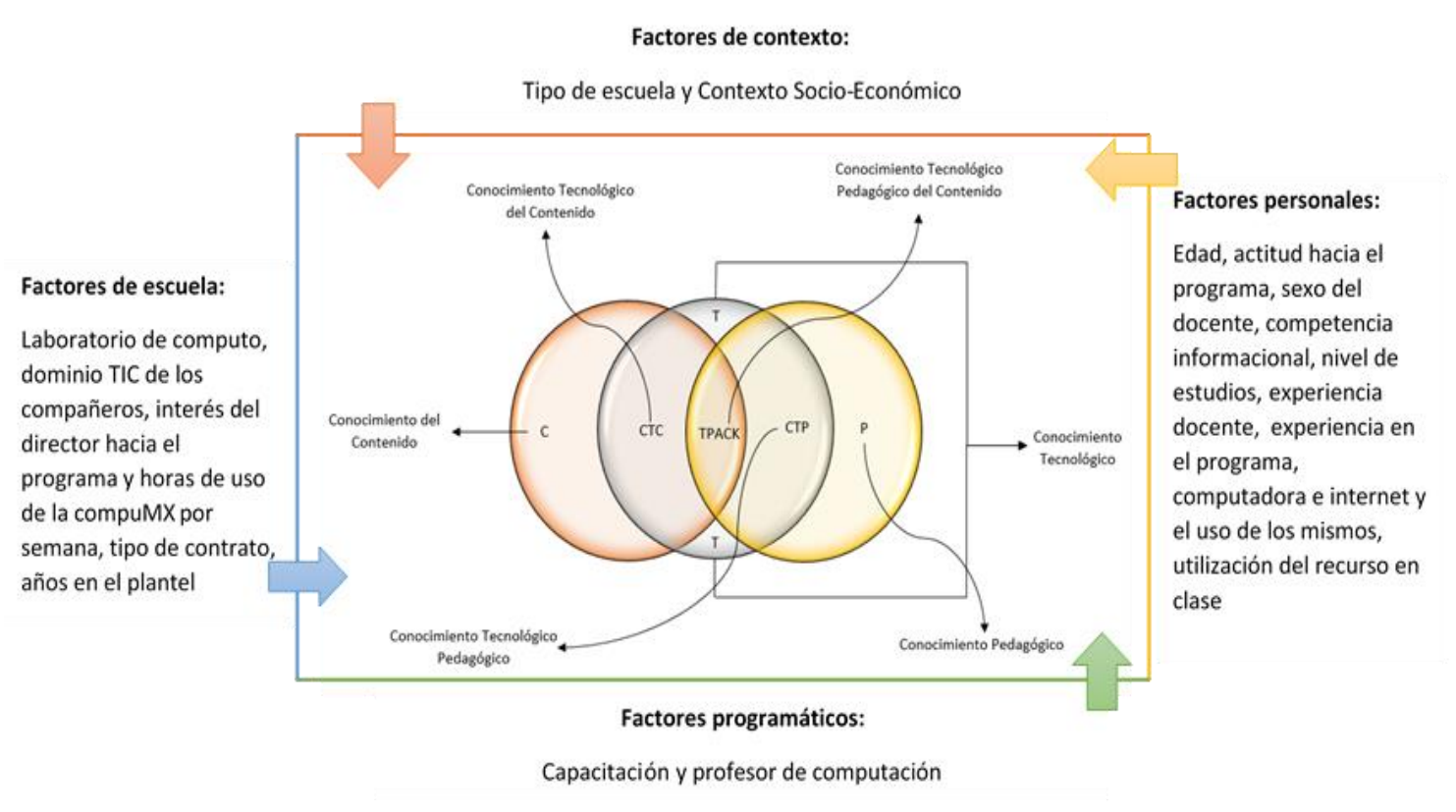

Figura 4. Modelo teórico de los factores relacionados con la integración de la tecnología en la educación

\subsection{Factores personales}

Se refieren a las características propias del docente y las acciones realizadas por el mismo. En la tabla $V$ se presentan los resultados de la aplicación de un modelo de regresión lineal múltiple, donde las variables que en mayor medida aportan en la integración de la tecnología en la educación (TPACK) son la actitud, el nivel de competencia informacional, el contar con Internet en casa y la experiencia en el programa, el conjunto de estas variables logra explicar el 51\% de dicha inclusión.

Tabla IV. Modelo de regresión de los factores personales

\begin{tabular}{l|c|c|c|c|c}
\hline Modelo & $\mathrm{B}$ & $\mathrm{ES} \mathrm{B}$ & $\boldsymbol{\beta}$ & $\boldsymbol{t}$ & $\boldsymbol{p}$ \\
\hline Actitud & 0.336 & 0.056 & 0.376 & 6.015 & 0.000 \\
Competencia informacional & 0.366 & 0.060 & 0.378 & 6.148 & 0.000 \\
Internet en casa & -0.229 & 0.094 & -0.146 & -2.435 & 0.016 \\
Años en el programa & 0.083 & 0.036 & 0.129 & 2.327 & 0.021 \\
\hline
\end{tabular}

Nota: $R^{2}=.510(N=203, p=.000)$

\section{Conclusiones}

Una de las bondades que ofrece el modelo tecno-educativo del TPACK es saber con qué conocimientos debería contar un docente en la actualidad; hacer uso de dicho modelo permite diseminar y medir esos conocimientos de manera aislada y en conjunto, de esta forma esta investigación ha encontrado que los conocimientos relacionados con el uso de la tecnología en la educación aún tienen un área importante de mejora; además, dicho déficit puede deberse a la falta de capacitación detectada o la falta de calidad de la misma, donde esta última se ha evidenciado en diversos estudios (Angulo, Torres y Valdés, 2013; Beltrán et al., 2015 a, b).

Por lo anterior, se recomienda desarrollar e implementar programas de capacitación que generen los conocimientos necesarios para el uso y dominio de la tecnología, así como que promuevan una actitud favorable hacia el empleo de la misma y el fortalecimiento de la competencia informacional, lo que sería benéfico hacer desde la formación inicial de los docentes (Badilla y Fuentes, 2014; Canales, 2014; Lucero, Meza y Aguirre, 2014; Salinas y Silva, 2014). 
Finalmente, una variable adicional que se identificó en el estudio fue el interés que muestra el director de la escuela respecto al uso que hace el docente de la tecnología que, si bien no resultó significativa, sí muestra información relevante en cuanto al apoyo que éste puede brindar a los profesores con el fin de lograr una mayor integración de la tecnología a las prácticas docentes. Por lo anterior, se recomienda continuar y ampliar investigaciones que compartan las mismas finalidades que este estudio considerando agentes educativos como los directivos escolares y los padres de familia, donde también se investiguen sus creencias y actitudes hacia las TIC en la educación (Ceretta y Picco, 2013; Lago, Mariotas y Amado, 2012); de esta forma pudiera realizarse un modelo de explicación que resultaría en un aporte significativo a la temática.

\section{Referencias}

Aguirre, D. (2014). Scientix: propuestas para insertar las tIC en la enseñanza de las ciencias. En V. Díaz y J. Muñoz (Coords). XVII Congreso Internacional EDUTEC: El hoy y el mañana junto a las TIC (pp. 752-763). Córdoba, España

Angeli, C. y Valanides, N. (2009). Epistemological and methodological issues for the conceptualización, development, and assessment of ICT-TPCK: Advances in technological pedagogical content knowledge (TPCK). Computers y Education, 52(1), 154-168. doi:10.1016/j.compedu.2008.07.006

Angulo, J., Torres, C., y Valdés, A. (2013). Necesidades de formación en el uso de las TIC en docentes de Sonora y Veracruz. En C. Torres, J. Angulo, A. Valdés y R. García (Eds.), Adopción tiC en docentes de nivel primaria (pp. 87-103). México: Pearson.

Archambault, L. y Barnett, J. (2010). Revisiting techological pedagogical content knowledge: Exploring the TPACK framework. Computers y Education, 55(4), 1656-1662. doi:10.1016/j.compedu.2010.07.009

Area, M. (2009). Introducción a la Tecnología Educativa. España: Universidad de la Laguna.

Badilla, M. y Fuentes, C. (2014). Integración pedagógica de la pizarra digital interactiva en la formación inicial docente. En J. Salinas y J. Silva (Eds.), Innovando con TIC en la formación inicial docente: Aspectos teóricos y casos concretos (pp. 57-70). Santiago: ENLACES.

Beltrán, J. A., García, R. I. y Ramírez, M. S. (2015a). Percepción docente sobre sus experiencias con el programa "Mi CompuMx". Memorias del Segundo Congreso Internacional de Innovación Educativa, México. Recuperado de http://hdl.handle.net/11285/575994

Beltrán, J. A., García, R. I. y Ramírez, M. S. (2015b). Usabilidad y apropiación del programa "Mi CompuMx" desde la perspectiva de los docentes de primaria. Memorias del XIII Congreso Nacional de Investigación Educativa, Chihuahua, México. Recuperado de http://hdl.handle.net/11285/575960

Cabero, J., Barroso, J., Cadena, A., Castaño, C., Cukieman, U. y Llorente, C. (2014). La formación del profesorado en TIC: Modelo TPACK. España: Universidad de Sevilla.

Canales, R. (2014). La inserción de las TIC para innovar en la formación inicial docente: caso Universidad de los Lagos en Chile. En J. Salinas y J. Silva (Eds.), Innovando con tIC en la formación inicial docente: aspectos teóricos y casos concretos (pp. 219-236). Santiago: ENLACES.

Ceretta, M. y Picco, P. (2013). La necesidad de definir un modelo de alfabetización en información para el Plan Ceibal. Revista TransInformação, 25(2), 127-133. Recuperado de

http://periodicos.puc-campinas.edu.br/seer/index.php/transinfo/article/view/1952

De Pablos, J., Colás, P. y González, T. (2009). Factores facilitadores de una la innovación con TIC en los centros escolares. Un análisis comparativo entre diferentes políticas educativas autonómicas. Revista de Educación, 352, 23-51. Recuperado de http://www.revistaeducacion.educacion.es/re352/re352 02.pdf 
Factores que influyen en la integración del Programa de Inclusión y Alfabetización Digital en la docencia en escuelas primarias

De Vellis, R. (2012). Scale development. Theory and applications. Estados Unidos: Sage.

Díaz, D. C., Rodríguez, M., Sánchez, W., Rivera, N. y Ramírez, M. S. (2015). Competencias digitales en el marco del programa Mi Compu Mx: estudio piloto en Colima, Sonora y Tabasco. Memorias del Segundo Congreso Internacional de Innovación Educativa, México. Recuperado de http://repositorio.itesm.mx/ortec/handle/11285/575398

Field, A. (2009). Discovering statistics using sPSS (3a. ed.). Londres: Sage.

García, R. I., Angulo, J. y Cuevas, O. (2015). Mi CompuMx: opinión de padres de familia, docentes y directivos sobre su aplicación y desarrollo. Memorias del XIII Congreso Nacional de Investigación Educativa, Chihuahua, México. Recuperado de http://hdl.handle.net/11285/575957

Gómez, M. (2006). Introducción a la metodología de la investigación científica. Argentina: Brujas.

González, F., Escoto, M. y Chávez, J. (2017). Estadística aplicada en Psicología y Ciencias de la salud. México: El Manual Moderno.

Kabakci, I., Ferhan, H., Kilicer, K., Naci, A., Birinci, G. y Askim, A. (2012). The development, validity and reliability of TPACK-deep: A Technological Pedagogical Content Knowledge Scale. Computers \&

Education,58(3), 964-977. Recuperado de doi:10.1016/j.compedu.2011.10.012

Lago, S., Mariotas, A. y Amado, S. (2012). Inclusión digital en la educación pública argentina. El programa Conectar Igualdad. Educación y Pedagogía, 24(62), 205-218. Recuperado de http://aprendeenlinea.udea.edu.co/revistas/index.php/revistaeyp/article/view/14204

Lázaro, J., Gisbert, M., González, J. y Camacho, M. (2014). La formación inicial y permanente en competencia digital del profesorado: una experiencia de colaboración interinstitucional. En V. Diaz y J. Muñoz (Coords.). XVII Congreso Internacional EDUTEC: El hoy y el mañana junto a las TIC (pp. 365-377). Córdoba, España.

Liu, S. (2011). Factors related to pedagogial beliefs of teachers and technology integración. Computers y Education, 56(4). Recuperado de doi:10.1016/j.compedu.2010.12.001

Llorente, C., Romero, R., Román P. y Barroso, J. (2014). Influencias de la capacitación del profesorado en tIC: conocimientos tecnológicos, pedagógicos y de contenidos. En V. Díaz y J. Muñoz (Coords). XVII Congreso Internacional EDUTEC: El hoy y el mañana junto a las TIC (pp. 411-420). Córdoba, España.

Lucero, I., Meza, S. y Aguirre, M. (2014). Las TIC en la formación inicial docente de un profesorado de física. En J. Silva y J. Salinas (Eds). Innovando con TIC en la formación inicial docente: aspectos teóricos y casos concretos (99-116). Santiago: ENLACES.

Martínez, C. y Cabello, M. (2012). La formación docente a través de las políticas públicas. La implementación de la educación virtual en México. Documento presentado en el I Congreso Virtual Internacional sobre Innovación Pedagógica y Praxis Educativa. Sevilla, España.

Mishra, P. y Koehler, M. J. (2006). Technological pedagogical content knowledge: A framework for teacher knowledge. Teachers College Record, 108(6), 1017-1054. doi:10.1111/j.1467-9620.2006.00684.x

Morales, V. (2013). Desarrollo de competencias digitales docentes en la educación básica. Revista Apertura, 5(1). Recuperado de http://www.udgvirtual.udg.mx/apertura/index.php/apertura3/article/view/367/307

Nogueira, F. (2014). Citizen education and technologies: potencial for teaching and implications on teacher education. En V. Diaz y J. Muñoz (Coords.), XVII Congreso Internacional EDUTEC: El hoy y el mañana junto a las TIC (pp. 1738-1747). Córdoba, España. 
One Laptop per Child. (2011). Principios pedagógicos. Recuperado de http://one.laptop.org/about/5-core-principles

Peeraer, J. y Van Petegem, P. (2010). Factors Influencing Integration of ICT in Higher Education in Vietnam. En Z. Abas, I. Jung y J. Luca (Eds.), Proceedings of Global Learn 2010 (pp. 916-924). Malasia: Association for the Advancement of Computing in Education.

Plan ceibal (2011). Qué es el Plan Ceibal. Recuperado de https://www.ceibal.edu.uy/es/institucional

Rangel, A. (enero, 2015). Competencias docentes digitales: propuesta de un perfil. Pixel-Bit. Revista de Medios y Educación, 46(1), 235-248. Recuperado de

https://recyt.fecyt.es/index.php/pixel/article/view/61622

Rivera, N., Mercado, M. y Ramírez, M. S. (2015). Aportes para curso masivo abierto a distancia (Mooc) de habilidades digitales para docentes: Investigación en el marco del programa Mi Compu.Mx. Memorias del XIII Congreso Nacional de Investigación Educativa, Chihuahua, México. Recuperado de http://hdl.handle.net/11285/575865

Rocha, M. A. y Ramírez, M. S. (2015). Los sujetos y objetos que inciden en el desarrollo de competencias digitales en el marco del programa Mi Compu Mx: Caso escuela rural de Colima. Memorias del XIII Congreso Nacional de Investigación Educativa, Chihuahua, México. Disponible en http://hdl.handle.net/11285/575878

Rodríguez, A., García, E., Ibáñez, R., González, J. y Heine, J. (2009). Las TIC en la educación superior: estudio de los factores intervinientes en la adopción de un LMS por docentes innovadores. Revista Latinoamericana de Tecnología Educativa, 8(1). Recuperado de https://relatec.unex.es/article/view/479

Salinas, J. y Silva, J. (2014). Innovación con tıc en formación inicial docente en Iberoamérica. En J. Salinas y J. Silva (Eds.), Innovando con TIC en la formación inicial docente: aspectos teóricos y casos concretos (pp. 1231). Santiago de Chile: ENLACES.

Schmidt, D. A., Baran, E., Thompson, A. D., Mishra, P., Koehler, M. J. y Shin, T. S. (2009). Technological pedagogical content knowledge (TPACK): The development and validation of an assessment instrument for preservice teachers. Journal of Research on Technology in Education, 42(2), 123-149.

doi:10.1080/15391523.2009.10782544

Secretaría de Educación Pública, (2013). Dotación de equipos de cómputo portátiles para niños de quinto y sexto grados de escuelas primarias públicas. Recuperado de https://www.slideshare.net/jssbltrn/dotacin-de-equipos-de-cmputo-porttiles-para-nios-de-quinto-y-sextogrados-de-escuelas-primarias-pblicas

Sing, C., Ling, J., Tsai, C., y Wee, L. (2011). Modeling primary school pre-service teachers' Technological Pedagogical Content Knowledge (TPACK) for meaningful learning with information and communication technology (ICT). Computers y Education, 57(1). Recuperado de https://www.sciencedirect.com/science/article/abs/pii/S0360131511000157

Staff Presidencia. (2013). Infografía sobre el programa micompu.mx. Recuperado de http://www.sct.gob.mx/despliega-noticias/article/avances-de-la-reforma-educativa/

Trucco, D. y Espejo, A. (2013). Principales determinantes de la integración de las TIC en el uso educativo: el caso del Plan Ceibal del Uruguay. Políticas sociales. 177. Recuperado de http://repositorio.cepal.org/handle/11362/6191

Valdés, Á., Angulo, J., Urías, M., García R. y Mortis, S. (2011). Necesidades de capacitación de docentes de educación básica en el uso de las TIC. Pixel-Bit. Revista de Medios y Educación, 39, 211-223. Recuperado de https://recyt.fecyt.es/index.php/pixel/article/view/61460 
Factores que influyen en la integración del Programa de Inclusión y Alfabetización Digital en la docencia en escuelas primarias

Valdés, A., Arreola, C., Angulo, J., Carlos, E., y García, R. (2011). Actitudes de docentes de educación básica hacia las TIC. Magis, Revista Internacional de Investigación en Educación, 3(6), 379-392. Recuperado de https://revistas.javeriana.edu.co/index.php/MAGIS/article/view/3546 